\title{
Master Symmetries of the XY Model
}

\author{
Huzihiro Araki * \\ Research Institute for Mathematical Sciences, Kyoto University, Kyoto 606, Japan
}

\section{Dedicated to Res Jost and Arthur Wightman}

\begin{abstract}
Master symmetries, found by Barouch and Fuchssteiner for a finite size $\mathrm{XY}$ model with the help of a computer program, are mathematically analyzed for an infinitely extended XY model by a rigorous operator algebraic method with an easy computation. The infinite family of commuting Hamiltonians and the master symmetries generating them form an infinite dimensional Lie group of automorphisms of a $C^{*}$-algebra of observables for the model.
\end{abstract}

\section{Introduction}

The one-dimensional XY-model in statistical mechanics of the spin 1/2 lattice system is known to be exactly solvable. One possible feature of an exactly solvable quantum model is the existence of a commuting family of explicitly describable operators (constants of motion or symmetry generators) which commute with the Hamiltonian of the model. Barouch and Fuchssteiner [7] found an interesting mechanism of creating such a commuting family, which will be quoted in detail in the next section.

Barouch and Fuchssteiner refers the proof to a computer computation. They give only the first few operators in the commuting family explicitly and no general explicit forms for the operators in an infinite family are given. The complicated expressions for the first few operators do not seem to suggest any general explicit form either. Thus we are not sure about the proof of the claim for the general operators in the infinite family, e.g. the proof of their commutativity.

Also Barouch and Fuchssteiner do not specify the boundary condition for the model. The expression for the master symmetry containing the number $j$ of the lattice site explicitly excludes the possibility of the periodic boundary condition. However the validity of the commutativity can be broken at the boundary for other boundary conditions.

\footnotetext{
* Supported in part by Mombusho International Scientific Research Program
} 
The aim of this paper is to present a more proper mathematical formulation, to provide a rigorous proof and to give manageable description of a general term (not just the first few operators in an infinite family) for master symmetries and commuting families derived from them.

We show that master symmetries and the commuting families derived from them (after multiplied by $i$ ) form an infinite dimensional Lie algebra of derivations defined on a common dense subalgebra $\mathfrak{A}_{0}$ in the $C^{*}$-algebra $\mathfrak{A}$, consisting of all local observables, that their closures are generators of one-parameter groups of automorphisms of $\mathfrak{A}$, forming an infinite-dimensional Lie group and that the subgroup generated by commuting families is abelian. Furthermore, it is found that each member of commuting families derived from all master symmetries under consideration is a finite linear combination of members of the commuting family generated by the basic master symmetry.

It is very likely that the abelian subgroup of automorphisms mentioned above (or its closure) is maximal abelian in the group of all automorphisms of $\mathfrak{A}$.

In Sect. 2, we formulate the main results as Theorems. Theorems 1 and 2 are essentially the claim of Barouch and Fuchssteiner [7], while Theorem 3 is new. While Theorems 1-3 are Lie algebra statements about derivations of the $C^{*}$ algebra of observables, Theorem 4 is a result about the exponentiation of the infinite Lie algebra into a group of automorphisms.

In Sect. 3, we review the $C^{*}$-algebraic method of solution for the onedimensional XY-model. In particular, the CAR algebra is introduced via the Jordan Wigner transformation from the spin algebra.

In Sect. 4, master symmetries and commuting families derived from them are explicitly computed as derivations on the CAR algebra.

In Sect. 5, the problem of going back from the CAR algebra to the algebra of observables is solved for derivations and the uniqueness is shown.

In Sect. 6, the exponentiation of derivations into automorphisms is carried out by the technique of Bogoliubov automorphisms of the CAR algebra and then by changing over to automorphisms of the spin algebra.

In Sect. 7, we collect results of the preceding sections into a proof of Theorems in Sect. 2. We also remark in Sect. 6 about the maximal abelian nature of the commuting family of automorphisms on the CAR algebra due to a theorem of Kishimoto [9] and conjecture the same for the spin algebra.

\section{Statement of Results}

We denote the lattice sites in one dimension by integers $\mathbf{Z}$, the Pauli spin operators at each lattice site $j \in \mathbf{Z}$ by $\sigma_{x}^{(j)}, \sigma_{y}^{(j)}$, and $\sigma_{z}^{(j)}$, the $C^{*}$-algebra of observables generated by them at all lattice sites $j \in \mathbf{Z}$ by $\mathfrak{A}$, and the subalgebra consisting of all finite polynomials of $\sigma$ 's by $\mathfrak{A}_{0}$.

The standard expression for the Hamiltonian of the XY-model is given by

$$
\begin{gathered}
H=-J \sum_{j \in \mathbf{Z}} H(j), \\
H(j)=(1+\gamma) \sigma_{x}^{(j)} \sigma_{x}^{(j+1)}+(1-\gamma) \sigma_{y}^{(j)} \sigma_{y}^{(j+1)}+2 \lambda \sigma_{z}^{(j)} .
\end{gathered}
$$


(In notation of [7], $-J(1+\gamma)=J_{x},-J(1-\gamma)=J_{y},-2 J \lambda=h$.) We interpret $H$ as the derivation

$$
\delta_{H} A=[H, A]=-J \sum_{j \in \mathbf{Z}}[H(j), A]
$$

for $A \in \mathfrak{A}_{0}$. Each term of the sum (2.1) defines an inner derivation, which vanishes for sufficiently large $j$ for any given $A \in \mathfrak{A}_{0}$ due to the commutativity of $\sigma$ 's at different lattice sites, i.e. $[H(j), A]$ vanishes if $j$ and $j+1$ are outside of supporting sites of $A$. (The supporting sites of an element $A$ may be defined as the set of all $j$ which appears in an explicit expression for $A$ in terms of $\sigma$ 's.) Therefore (2.3) is actually a finite sum and belongs to $\mathfrak{A}_{0}$ for any $A \in \mathfrak{A}_{0}$.

In this note, we explicitly exclude the case $(\lambda, \gamma)=(0, \pm 1)$ (the case of onedimensional Ising model).

We consider another derivation of a similar nature given by

$$
\begin{gathered}
S=\sum_{j \in \mathbf{Z}} S(j), \\
S(j)=\sigma_{x}^{(j)} \sigma_{y}^{(j+1)}-\sigma_{y}^{(j)} \sigma_{x}^{(j+1)} .
\end{gathered}
$$

We use the following "master symmetry" in the sense of a derivation on $\mathfrak{A}_{0}$,

$$
P=-\sum_{j \in \mathbf{Z}} P(j), \quad P(j)=(j+(1 / 2)) H(j)-\lambda \sigma_{z}^{(j)} .
$$

This is related to the first master symmetry $M_{0}$ of Barouch and Fuchssteiner ((3.2) and (3.7) in [7], where (3.7) should read $\lambda=-(1 / 2) h$ ) via

$$
M_{0}=J P-(1 / 2) H,
$$

which produces the same family of commuting "Hamiltonians" as $P$ except for the constant coefficient $J$. We note that $P(j)$ of (2.6) can be obtained from $H(j)$ of $(2.2)$ by multiplying the terms $\sigma_{x}^{(j)} \sigma_{x}^{(j+1)}$ and $\sigma_{y}^{(j)} \sigma_{y}^{(j+1)}$ by $(j+(1 / 2))$ and the term $\sigma_{z}^{(j)}$ by $j$, the purpose of the $\sigma_{z}^{(j)}$ term being just to cancel (1/2) in the coefficient of $H(j)$ for the $\sigma_{z}^{(j)}$ term.

The derivation $P$ is called a master symmetry because of the following property.

Theorem 1. Starting with $H_{0}=H$ and $S_{0}=S$, the derivations $H_{n}$ and $S_{n}$ on $\mathfrak{A}_{0}$ are defined recursively by

$$
\begin{aligned}
H_{(n+1)} & =i\left[P, H_{n}\right], \\
S_{(n+1)} & =i\left[P, S_{n}\right],
\end{aligned}
$$

$n=0,1,2, \ldots$. Then for any $j, k \in \mathbf{N} \cup\{0\}$,

$$
\left[H_{j}, H_{k}\right]=\left[H_{j}, S_{k}\right]=\left[S_{j}, S_{k}\right]=0 .
$$

More explicit description of $H_{n}$ and $S_{n}$ as well as the proof will be given later. We note that the commutator of derivations are defined, for example, by

$$
\delta_{H_{n+1}}(A)=i\left(\delta_{P}\left\{\delta_{H_{n}}(A)\right\}-\delta_{H_{n}}\left\{\delta_{P}(A)\right\}\right) .
$$


By (2.1) and (2.6), we have for $A \in \mathfrak{A}_{0}$

$$
\begin{aligned}
\delta_{H_{1}}(a) & =i J \sum_{j, k}\{[P(j),[H(k), A]]-[H(k),[P(j), A]]\} \\
& =i J \sum_{j, k}[[P(j), H(k)], A],
\end{aligned}
$$

where the last equality is by Jacobi identity. This will be denoted by

$$
H_{1}=i J \sum_{j, k}[P(j), H(k)] \text {. }
$$

Similar expressions can be written down for other $H_{n}$ and $S_{n}$.

We define $g_{0}$ to be the set of all finite linear combination of $H_{n}, n=0,1,2, \ldots$ and $S_{n}, n=0,1,2, \ldots$ with real coefficients. Addition of any element of $\mathfrak{g}_{0}$ to the master symmetry $P$ does not change $H_{n}$ and $S_{n}$ defined above due to the abelian nature of $\mathfrak{g}_{0}$. In particular $M_{0}$ of Barouch and Fuchssteiner and our $P$ produce the same $H_{n}$ and $S_{n}$ except for a constant coefficient $J^{n}$.

In the same spirit, we define another master symmetry $P_{1}$ :

$$
\begin{gathered}
P_{1}=-\sum_{j \in \mathbf{Z}} P_{1}(j) \\
P_{1}(j)=(j+1)\left\{(1+\gamma) \sigma_{x}^{(j)} \sigma_{z}^{(j+1)} \sigma_{x}^{(j+2)}+(1-\gamma) \sigma_{y}^{(j)} \sigma_{z}^{(j+1)} \sigma_{y}^{(j+2)}\right\} \\
-2 j \sigma_{z}^{(j)}-(2 j+1) \lambda\left(\sigma_{x}^{(j)} \sigma_{x}^{(j+1)}+\sigma_{y}^{(j)} \sigma_{y}^{(j+1)}\right) .
\end{gathered}
$$

This differs from the second master symmetry $M_{1}$ of Barouch and Fuchssteiner by a constant coefficient and an addition of elements of $g_{0}$ :

$$
M_{1}=4 J^{2} P_{1}+2 J^{2} S_{1}-4 J \lambda H \text {. }
$$

We now define an infinite family of master symmetries recursively by

$$
P_{j+1}=i\left[P, P_{j}\right], \quad j=1,2, \ldots
$$

As before, the $P_{j}(j=l, 2, \ldots)$ are understood as derivations on $\mathfrak{A}_{0}$.

Each of $P_{j}$ produces a commuting family of derivations on $\mathfrak{U}_{0}$, which commute mutually for different $j$ :

$$
\begin{array}{cl}
H_{k+1, j}=i\left[P_{j}, H_{k, j}\right], & H_{0, j}=H, \\
S_{k+1, j}=i\left[P_{j}, S_{k, j}\right], & S_{0, j}=S .
\end{array}
$$

Here $k=0,1,2, \ldots$

We extend the notation of (2.17) and (2.18) for $j=0$ with $P_{0}=P$, so that $H_{k, 0}=H_{k}, S_{k, 0}=S_{k}$. We have

Theorem 2. For all $k, j, l, m=0,1,2, \ldots$,

$$
\left[H_{k, j}, H_{l, m}\right]=\left[H_{k, j}, S_{l, m}\right]=\left[S_{k, j}, T_{l, m}\right]=0 .
$$

We note that $M_{j}$ in [7] and $4 J^{j+1} P_{j}, j=1,2, \ldots$ differ by an element of $g_{0}$ for each $j$ and hence produce the same family of $H$ 's and $S$ 's as $H$ 's and $T$ 's of Barouch and Fuchssteiner up to constant coefficients. Thus Theorem 2 is the claim by Barouch and Fuchssteiner, for which we give a straightforward proof. Actually we have the following stronger result, which proves Theorem 2 in view of Theorem 1. 
Theorem 3. For all $k, j=0,1,2, \ldots$,

$$
H_{k, j} \in \mathfrak{g}_{0}, \quad S_{k, j} \in \mathfrak{g}_{0} .
$$

We now describe our result about integrating (or exponentiating) these derivations. Let $g_{1}$ be the set of all finite real linear combinations of $P_{k}$, $k=0,1,2, \ldots$ and elements of $\mathfrak{g}_{0}$.

Theorem 4. For any $X \in \mathfrak{g}_{1}$, the closure of $i X$ is the generator of a one-parameter group of automorphisms of $\mathfrak{U}$, which will be denoted as $e^{i X}$. For $X, Y \in \mathfrak{g}_{0}, e^{i X}$ and $e^{i Y}$ commute.

Actually we can describe the group generated by $e^{i X}, X \in \mathfrak{g}_{1}$ in more detail. This will be a subject of the forthcoming paper.

\section{The Method of Solution}

\section{Introduction of CAR-Algebra}

We now quote the operator algebraic method of solution for the XY-model $[3,4,5]$.

We introduce two involutive automorphisms of $\mathfrak{A}$ defined as follows:

$$
\begin{gathered}
\Theta(A)=\lim _{N \rightarrow \infty}\left(\prod_{j=-N}^{N} \sigma_{z}^{(j)}\right) A\left(\prod_{j=-N}^{N} \sigma_{z}^{(j)}\right), \\
\Theta_{-}(A)=\lim _{N \rightarrow \infty}\left(\prod_{j=-N}^{0} \sigma_{z}^{(j)}\right) A\left(\prod_{j=-N}^{0} \sigma_{z}^{(j)}\right) .
\end{gathered}
$$

More explicitly, they can be defined by the following action on the generators of $\mathfrak{A}$ :

$$
\begin{gathered}
\Theta\left(\sigma_{x}^{(j)}\right)=-\sigma_{x}^{(j)}, \quad \Theta\left(\sigma_{y}^{(j)}\right)=-\sigma_{y}^{(j)}, \quad \Theta\left(\sigma_{z}^{(j)}\right)=\sigma_{z}^{(j)} . \\
(j=0, \pm 1, \pm 2, \ldots) . \\
\Theta_{-}\left(\sigma_{\alpha}^{(j)}\right)=\left\{\begin{array}{lll}
\sigma_{\alpha}^{(j)} & \text { if } j \geqq 1 \\
\Theta\left(\sigma_{\alpha}^{(j)}\right) & \text { if } & j \leqq 0 . \\
(\alpha=x, y, z) .
\end{array}\right.
\end{gathered}
$$

Let $\hat{\mathfrak{U}}$ be the crossed product of $\mathfrak{U}$ by the action $\Theta_{-}^{n}$ of $n \in \mathbf{Z}_{2}$. More explicitly, $\hat{\mathfrak{A}}$ is the direct sum

$$
\hat{\mathfrak{U}}=\mathfrak{A}+T \mathfrak{U},
$$

where the element $T$ of $\hat{\mathfrak{U}}$ satisfies

$$
T^{2}=1, \quad T^{*}=T, \quad T A T=\Theta_{-}(A)
$$

for all $A \in \mathfrak{A}$. The automorphisms $\Theta$ and $\Theta_{-}$of $\mathfrak{A}$ can be extended to automorphisms of $\hat{\mathfrak{A}}$ (again written by the same letters $\Theta$ and $\Theta_{-}$) satisfying $\Theta(T)$ $=\Theta_{-}(T)=T$.

The algebras $\mathfrak{A}, \mathfrak{U}_{0}$, and $\hat{\mathfrak{U}}$ are decomposed into a sum of $\Theta$-even and $\Theta$-odd parts via the following elementwise decomposition:

$$
A=A_{+}+A_{-}, \quad A_{ \pm}=2^{-1}(A \pm \Theta(A)),
$$


where $\Theta\left(A_{ \pm}\right)= \pm A_{ \pm}$by definition. Accordingly,

$$
\mathfrak{U}=\mathfrak{A}_{+}+\mathfrak{A}_{-}, \quad \mathfrak{A}_{0}=\mathfrak{A}_{0+}+\mathfrak{A}_{0-}, \quad \hat{\mathfrak{U}}=\hat{\mathfrak{U}}_{+}+\hat{\mathfrak{U}}_{-} .
$$

We define the following $C^{*}$-subalgebra of $\hat{\mathfrak{U}}$.

$$
\mathfrak{A C A R}^{\mathrm{CAR}}=\mathfrak{U}_{+}+T \mathfrak{A}_{-} .
$$

It is generated by the so-called Jordan-Wigner transform of $\sigma$ 's,

where

$$
\begin{aligned}
& c_{j}=T S_{j}\left(\sigma_{x}^{(j)}-i \sigma_{y}^{(j)}\right) / 2, \\
& c_{j}^{*}=T S_{j}\left(\sigma_{x}^{(j)}+i \sigma_{y}^{(j)}\right) / 2,
\end{aligned}
$$

These operators satisfy the canonical anticommutation relations (CAR):

$$
\left[c_{j}, c_{k}\right]_{+}=\left[c_{j}^{*}, c_{k}^{*}\right]_{+}=0, \quad\left[c_{j}, c_{k}^{*}\right]_{+}=\delta_{j k},
$$

where $[A, B]_{+}=A B+B A$ and $\delta_{j k}$ is 0 for $j \neq k$ and 1 for $j=k$.

The automorphism $\Theta_{-}$of $\hat{\mathfrak{A}}$ leaves $\mathfrak{A}^{\mathrm{CAR}}$ invariant (as a set) and $\hat{\mathfrak{U}}$ can also be viewed as the crossed product of $\mathfrak{A}^{\mathrm{CAR}}$ by the $\Theta_{-}$action of $\mathbf{Z}_{2}$. Generators of $\mathfrak{A}$ can be expressed in terms of creation and annihilation operators $c_{j}^{*}$ and $c_{j}$ and $T$ as follows:

$$
\sigma_{j}^{(z)}=2 c_{j}^{*} c_{j}-1, \quad \sigma_{x}^{(j)}=T S_{j}\left(c_{j}+c_{j}^{*}\right), \quad \sigma_{y}^{(j)}=T S_{j} i\left(c_{j}-c_{j}^{*}\right) .
$$

Again, $\mathfrak{A}^{\mathrm{CAR}}$ can be split into $\Theta$-even and $\Theta$-odd parts:

$$
\begin{gathered}
\mathfrak{I}^{\mathrm{CAR}}=\mathfrak{H}_{+}^{\mathrm{CAR}}+\mathfrak{H}_{-}^{\mathrm{CAR}}, \\
\mathfrak{U}_{+}^{\mathrm{CAR}}=\mathfrak{U}_{+}, \quad \mathfrak{A}_{-}^{\mathrm{CAR}}=T \mathfrak{U}_{-} .
\end{gathered}
$$

2. Selfdual Formulation of CAR and Bogoliubov Automorphisms $[1,6]$ We introduce the complex Hilbert space

$$
\mathscr{H}=l_{2}(\mathbf{Z}) \oplus l_{2}(\mathbf{Z}) .
$$

An element $h$ of $\mathscr{H}$ consists of two elements $f$ and $g$ of $l_{2}(\mathbf{Z})$, denoted by

$$
h=\left(\begin{array}{l}
f \\
g
\end{array}\right)
$$

instead of $h=f \oplus g$. Each $f \in l_{2}(\mathbf{Z})$ can be described by its components $f_{j}, j \in \mathbf{Z}$. We denote

$$
B(h)=\sum f_{j} c_{j}^{*}+\sum g_{j} c_{j},
$$

which converges in the $C^{*}$-norm of $\mathfrak{Q}^{\mathrm{CAR}}$. We introduce the antilinear involution $\Gamma$ on $\mathscr{H}$ by

$$
\Gamma\left(\begin{array}{l}
f \\
g
\end{array}\right)=\left(\begin{array}{l}
\bar{g} \\
\bar{f}
\end{array}\right),
$$


where the bar in $\bar{f}$ and $\bar{g}$ denotes the componentwise complex conjugation. Then $\{B(h)\}$ generates $\mathfrak{Y}^{\mathrm{CAR}}$ and satisfies the following selfdual form of CAR:

$$
\begin{gathered}
{\left[B\left(h_{1}\right)^{*}, B\left(h_{2}\right)\right]_{+}=\left(h_{1}, h_{2}\right) 1,} \\
B(h)^{*}=B(\Gamma h) .
\end{gathered}
$$

Here we are using the notation of physicists for the inner product:

$$
\left(h_{1}, h_{2}\right)=\left(f_{1}, f_{2}\right)+\left(g_{1}, g_{2}\right)=\sum_{j}\left(\bar{f}_{1 j} f_{2 j}+\bar{g}_{1 j} g_{2 j}\right) .
$$

For any unitary operator $U$ commuting with $\Gamma$, there exists the unique automorphism of $\mathfrak{U}^{\text {CAR }}$, denoted by $\alpha_{U}$ and called a Bogoliubov automorphism, satisfying

$$
\alpha_{U}(B(h))=B(U h) .
$$

Examples are $\Theta=\alpha_{-1}$ for $U=-1$ and $\Theta_{-}=\alpha_{\theta_{-}}$where

\section{XY-Model Hamiltonian}

$$
\left(\theta_{-} f\right)_{j}= \begin{cases}f_{j} & j \geqq 1 \\ -f_{j} & j \leqq 0\end{cases}
$$

The operator $H(j)$ given by (2.2) belongs to $\mathfrak{A}_{+}=\mathfrak{A}_{+}^{\text {CAR }}$ and can be written as a quadratic expression of $c_{j}, c_{j+1}, c_{j}^{*}$ and $c_{j+1}^{*}$ up to a constant term which does not count as a derivation:

$$
H(j)=2\left(c_{j} c_{j+1}^{*}+c_{j+1} c_{j}^{*}\right)+2 \gamma\left(c_{j} c_{j+1}+c_{j+1}^{*} c_{j}^{*}\right)+4 \lambda c_{j}^{*} c_{j}-2 \lambda .
$$

The derivation (2.3) can now be explicitly written:

$$
\begin{gathered}
\delta_{H}(B(h))=B(2 J \mathbf{K} h), \\
\mathbf{K}=\left(\begin{array}{ll}
U+U^{*}-2 \lambda, & \gamma\left(U-U^{*}\right) \\
-\gamma\left(U-U^{*}\right), & -\left(U+U^{*}-2 \lambda\right)
\end{array}\right) .
\end{gathered}
$$

Here the $2 \times 2$ matrix notation for the operator $\mathbf{K}$ on $\mathscr{H}$ corresponds to the 2 components notation (3.6) for elements $h$ of $K, U$ and $U^{*}$ are shift operators on $l_{2}(\mathbf{Z})$ to the left and to the right:

$$
(U f)_{j}=f_{j+1}, \quad\left(U^{*} f\right)_{j}=f_{j-1} .
$$

The operator $\mathbf{K}$ is bounded, acting on $\mathscr{H}$ and satisfies

$$
\mathbf{K}^{*}=\mathbf{K}, \quad \Gamma \mathbf{K}=-\mathbf{K} \Gamma .
$$

The derivation $\delta_{H}$ exponentiate to a one-parameter group of automorphisms $\left(\alpha_{H}\right)_{t}=e^{i \delta_{H}}, t \in \mathbf{R}$, of $\mathfrak{A}^{\mathrm{CAR}}$, explicitly given as Bogoliubov automorphisms:

$$
\left(\alpha_{H}\right)_{t}=\alpha_{U(t)}, \quad U(t)=\exp i(2 J \mathbf{K} t) .
$$

In subsequent computations, it is advantageous to introduce the following Fourier transform. For each $f \in l_{2}(Z)$ and $h \in \mathscr{H}$, we define functions of $\theta \in \mathbf{T}$ $=\mathbf{R} / 2 \pi \mathbf{N}$ as follows:

$$
f(\theta)=\sum_{n \in \mathbf{Z}} e^{i n \theta} f_{n}, \quad h(\theta)=\sum_{n \in \mathbf{Z}} e^{i n \theta} h_{n} .
$$


Here $f(\theta)$ is a complex number and $h(\theta)$ is a 2 dimensional vector for each $\theta$. The inverse relations are

$$
\begin{aligned}
& f_{n}=(2 \pi)^{-1} \int_{0}^{2 \pi} e^{-i n \theta} f(\theta) d \theta, \\
& h_{n}=(2 \pi)^{-1} \int_{0}^{2 \pi} e^{-i n \theta} h(\theta) d \theta .
\end{aligned}
$$

The operator $\mathbf{K}$ is diagonalized by this transformation.

$$
\begin{gathered}
(\mathbf{K} h)(\theta)=\hat{K}(\theta) h(\theta), \\
\hat{K}(\theta)=2\left(\begin{array}{ll}
\cos \theta-\lambda & -i \gamma \sin \theta \\
i \gamma \sin \theta & \lambda-\cos \theta
\end{array}\right) .
\end{gathered}
$$

We note that

$$
\widehat{K}(\theta)^{2}=k^{2}(\theta) 1, \quad k(\theta)=2\left[(\lambda-\cos \theta)^{2}+\gamma^{2} \sin ^{2} \theta\right]^{1 / 2} .
$$

We also note that

$$
(\Gamma h)(\theta)=\left(\overline{\frac{g(-\theta)}{f(-\theta)}}\right) \text { for } \quad h(\theta)=\left(\begin{array}{l}
f(\theta) \\
g(\theta)
\end{array}\right) .
$$

\section{Master Symmetries - Lie Algebra Computation}

All the derivations introduced in Sect. 2 are infinite sums of inner derivations by elements of $\mathfrak{A}_{+}=\mathfrak{U}_{+}^{\mathrm{CAR}}$, and hence we compute them first for $\mathfrak{Q}^{\mathrm{CAR}}$, using notation of the preceding section.

First, $S(j)$ of $(2.5)$ can be written as

$$
S(j)=2 i\left(c_{j+1}^{*} c_{j}-c_{j}^{*} c_{j+1}\right) .
$$

Therefore the derivation $\delta_{S}(A)=\Sigma[S(j), A]$ for $A=B(h)$ is given by

$$
\begin{gathered}
\delta_{S}(B(h))=B(\mathbf{S} h), \\
\mathbf{S}=2 i\left(\begin{array}{cc}
U^{*}-U & 0 \\
0 & U^{*}-U
\end{array}\right), \quad \hat{S}(\theta)=-4 \sin \theta \mathbf{1} .
\end{gathered}
$$

Next, $P(j)$ of (2.6) can be written as

$$
P(j)=(j+(1 / 2)) H(j)-\lambda\left(2 c_{j}^{*} c_{j}-1\right),
$$

where $H(j)$ is given by (3.23). Therefore $\delta_{P}(A)=-\Sigma[P(j), A]$ for $A=B(h)$ is given by

$$
\begin{gathered}
\delta_{P}(B(h))=B(\mathbf{P} h), \\
\mathbf{P}=2\left(\begin{array}{cc}
U_{1}+U_{1}^{*}-2 \lambda \delta, & \gamma\left(U_{1}-U_{1}^{*}\right) \\
\gamma\left(U_{1}^{*}-U_{1}\right), & -\left(U_{1}+U_{1}^{*}-2 \lambda \delta\right)
\end{array}\right),
\end{gathered}
$$

where $U_{1}$ and $\delta$ are defined by

$$
\begin{gathered}
(\delta h)_{j}=j h_{j}, \quad(\delta h)(\theta)=(-\mathrm{id} / d \theta) h(\theta)=-i h^{\prime}(\theta), \\
U_{1}=(\delta+(1 / 2)) U, \quad U_{1}^{*}=U^{*}(\delta+(1 / 2))=(\delta-(1 / 2)) U^{*} .
\end{gathered}
$$


Note that $\delta U=U(\delta-1),(\delta-1) U^{*}=U^{*} \delta$. The last term in (4.4) serves the purpose of canceling out the (1/2) term for $-2 \lambda$ in $H(j)$. The above expression for $\mathbf{P}$ can be written in a compact way

$$
\mathbf{P}=\delta \mathbf{K}+\mathbf{K} \delta,
$$

because $2 U_{1}=\delta U+U \delta$ and $2 U_{1}^{*}=\delta U^{*}+U^{*} \delta$, where $\mathbf{K}$ is given by (3.24b).

A basic property of $\mathbf{P}$ is the following commutation relation:

$$
i[\mathbf{P}, \mathbf{K}]=i\left[\delta, \mathbf{K}^{2}\right]=2 k k^{\prime} \mathbf{1}
$$

due to (3.31), where $k^{\prime}$ is the multiplication of the derivative $k^{\prime}(\theta)$ :

$$
\left(k^{\prime} h\right)(\theta)=k^{\prime}(\theta) h(\theta) \text {. }
$$

Computing in $\theta$-representation of the test function space $\mathscr{H}$, we obtain

$$
\begin{aligned}
i[\mathbf{P}, a \mathbf{1}] & =2 a^{\prime} \mathbf{K}, \\
i[\mathbf{P}, b \mathbf{K}]= & i[P, b] K+b i[P, K] \\
= & \left(2 b^{\prime} k^{2}+2 b k k^{\prime}\right) \mathbf{1}=2 k(d / d \theta)(k b) \mathbf{1}
\end{aligned}
$$

for any multiplication operators $a$ and $b$ in $\theta$-representations. By repeating these computations, we obtain the following description of the derivations $H_{j}$ on $\mathfrak{Q}^{\text {CAR }}$.

\section{Proposition 4.1.}

$$
\delta_{\mathbf{H}_{j}}(B(h))=B\left(\mathbf{H}_{j} h\right),
$$

where $\mathbf{H}_{j}$ is the multiplication of the following expressions in $\theta$-representation of $\mathscr{H}$ :

$$
\hat{H}_{j}(\theta)= \begin{cases}h_{j}(\theta) 1 & \text { for odd } j \\ h_{j}(\theta) K(\theta) & \text { for even } j .\end{cases}
$$

The functions $h_{j}$ satisfy

$$
h_{2 n}=2 D h_{2 n-1}, \quad h_{2 n+1}=2 k D k h_{2 n},
$$

where $D=(d / d \theta)$. With the initial condition $h_{0}=2 J$, they are given by

where $n=0,1,2, \ldots$.

$$
\begin{gathered}
h_{2 n}=2^{2 n+1} J(D \cdot k)^{2 n} \mathbf{1}, \\
h_{2 n+1}=2^{2 n+2} J k \cdot(D \cdot k)^{2 n+1} \mathbf{1},
\end{gathered}
$$

Here the product $D \cdot k$ is the product of the differentiation operator $D=(d / d \theta)$ and the multiplication operator $k$, and it does not mean the differentiation of the function $k(\theta)$ alone. The initial condition is by (3.24a).

Similarly, we obtain the following.

\section{Proposition 4.2.}

$$
\delta_{\mathbf{S}_{j}}(B(h))=B\left(\mathbf{S}_{j} h\right),
$$

where $\mathbf{S}_{j}$ is the multiplication of the following expression in $\theta$-representation of $\mathscr{H}$ :

$$
\hat{S}_{j}(\theta)= \begin{cases}s_{j}(\theta) \mathbf{1} & \text { for evenj } \\ s_{j}(\theta) \hat{K}(\theta) & \text { for odd } j\end{cases}
$$


The functions $s_{j}$ satisfy

$$
s_{2 n}=2 k D k s_{2 n-1}, \quad s_{2 n+1}=2 D s_{2 n} .
$$

With the initial condition $s_{0}(\theta)=-4 \sin \theta$, they are given by

$$
\begin{gathered}
s_{2 n}=-2^{2 n+2}(k D)^{2 n} \sin \theta, \\
s_{2 n+1}=-2^{2 n+3} D(k D)^{2 n} \sin \theta,
\end{gathered}
$$

where $n=0,1,2, \ldots$.

The initial condition is given by (4.3).

We now compute the second master symmetry $P_{1}$. We have

$$
\begin{aligned}
P_{1}(j)= & -2(j+1)\left\{c_{j+2}^{*} c_{j}+c_{j}^{*} c_{j+2}+\gamma\left(c_{j}^{*} c_{j+2}^{*}+c_{j+2} c_{j}\right)\right\} \\
& -4 j c_{j}^{*} c_{j}+2 j+2 \lambda(2 j+1)\left(c_{j+1}^{*} c_{j}+c_{j}^{*} c_{j+1}\right) .
\end{aligned}
$$

Therefore

$$
\delta_{P_{1}}(B(h))=B\left(\mathbf{P}_{1} h\right),
$$

$$
\begin{aligned}
\mathbf{P}_{1} & =2\left(\begin{array}{cc}
U^{* 2}(\delta+1)+U^{2}(\delta-1)+2 \delta-\lambda\left(U^{*}(2 \delta+1)+U(2 \delta-1)\right), & \gamma\left(U^{2}(\delta-1)-U^{* 2}(\delta+1)\right) \\
\gamma\left(U^{* 2}(\delta+1)-U^{2}(\delta-1)\right), & -U^{* 2}(\delta+1)-U^{2}(\delta-1)-2 \delta+\lambda\left(U^{*}(2 \delta+1)+U(2 \delta-1)\right)
\end{array}\right) \\
& =(1 / 2)\left\{\left(U+U^{*}\right) \mathbf{P}+\mathbf{P}\left(U+U^{*}\right)\right\} .
\end{aligned}
$$

We can now compute all members of the infinite family of master symmetries $P_{j}$ :

\section{Proposition 4.3.}

$$
\begin{gathered}
\delta_{P_{j}}(B(h))=B\left(\mathbf{P}_{j} h\right), \\
\mathbf{P}_{j}=\mathbf{L}_{j} \mathbf{P}+\mathbf{P L}_{j},
\end{gathered}
$$

where the operators $\mathbf{L}_{j}$ satisfy the recursive relation

$$
\mathbf{L}_{j+1}=i\left[\mathbf{P}, \mathbf{L}_{j}\right], \quad j=1,2, \ldots .
$$

They are the multiplication of the following expressions in $\theta$-representation:

$$
\hat{L}_{j}(\theta)= \begin{cases}p_{j}(\theta) 1 & \text { for odd } j \\ p_{j}(\theta) K & \text { for even } j,\end{cases}
$$

where the functions $p_{j}$ satisfy the recursive relations

$$
\begin{gathered}
p_{2 n+1}=2(k D k) p_{2 n}, \\
p_{2 n}=2 p_{2 n-1}^{\prime} .
\end{gathered}
$$

With the initial condition $p_{1}=\cos \theta$, they are given by

$$
\begin{gathered}
p_{2 n+1}=2^{2 n}(k D)^{2 n} \cos \theta, \\
p_{2 n}=-2^{2 n-1}(D k)^{2 n-2} \sin \theta .
\end{gathered}
$$


The initial condition is due to (4.24), in which $\left(U+U^{*}\right) / 2$ is the multiplication of $\cos \theta$ in $\theta$-representation.

\section{Proposition 4.4.}

$$
\delta_{\mathbf{H}_{k, j}}(B(h))=B\left(\mathbf{H}_{k, j} h\right),
$$

where $\mathbf{H}_{k, j}$ is the multiplication of the following expression in $\theta$-representation:

$$
\hat{H}_{k, j}(\theta)= \begin{cases}h_{k, j}(\theta) 1 & \text { for odd } j \text { and odd } k, \\ h_{k, j}(\theta) K & \text { otherwise }\end{cases}
$$

$k=1,2, \ldots, j=1,2, \ldots$. The functions $h_{k, j}$ satisfy the following recursion relations:

$$
\begin{gathered}
h_{2 n+1, j}=4\left(p_{j} k D k\right) h_{2 n, j} \quad \text { for odd } j, \\
h_{2 n+2, j}=4\left(p_{j} D\right) h_{2 n+1, j} \quad \text { for odd } j, \\
h_{l+1, j}=4\left(p_{j} k D k\right) h_{l, j} \quad \text { for even } j,
\end{gathered}
$$

where $n=0,1,2, \ldots, l=0,1,2, \ldots$ With the initial condition $h_{0, j}=2 J$ (a constant function), they are given by

$$
\begin{gathered}
h_{2 n+1, j}=\left(4 p_{j} k D\right)^{2 n+1} 2 J k \quad \text { for odd } j, \\
h_{2 n, j}=4 p_{j} D\left(4 p_{j} k D\right)^{2 n-1} 2 J k \quad \text { for odd } j, \\
h_{l, j}=\left(4 p_{j} k D k\right)^{l} 2 J \quad \text { for even } j .
\end{gathered}
$$

Proposition 4.5.

$$
\delta_{S_{k, j}}(B(h))=B\left(\mathbf{S}_{k, j} h\right),
$$

where $\mathbf{S}_{k, j}$ is the multiplication of the following expression in $\theta$-representation:

$$
\hat{S}_{k, j}(\theta)= \begin{cases}s_{k, j}(\theta) K & \text { for odd } j \text { and odd } k, \\ s_{k, j}(\theta) 1 & \text { otherwise, }\end{cases}
$$

$k=1,2, \ldots, j=1,2, \ldots$. The functions $s_{k, j}$ satisfy the following recursion relations:

$$
\begin{array}{cc}
s_{2 n, j}=4 p_{j} k D k s_{2 n-1, j} & \text { for odd } j, \\
s_{2 n+1, j}=4 p_{j} D s_{2 n, j} & \text { for odd } j, \\
s_{l+1, j}=4 p_{j} k^{2} D s_{l, j} & \text { for even } j .
\end{array}
$$

With the initial condition $s_{0, j}=-4 \sin \theta$, they are given by

$$
\begin{aligned}
s_{2 n, j} & =-4^{2 n+1}\left(p_{j} k D\right)^{2 n} \sin \theta \quad \text { for odd } j, \\
s_{2 n+1, j} & =-4^{2 n+2} p_{j} D\left(p_{j} k D\right)^{2 n} \sin \theta \quad \text { for odd } j, \\
s_{l, j} & =-4^{l+1}\left(p_{j} k^{2} D\right)^{l} \sin \theta \quad \text { for even } j .
\end{aligned}
$$

\section{Extension of Derivations to $\mathfrak{A}$}

For any finite interval $I$ of integers $\mathbf{Z}$, we define $\hat{\mathfrak{U}}(I)$ to be the subalgebra of $\hat{\mathfrak{A}}$ generated by $\sigma_{x}^{(j)}, \sigma_{y}^{(j)}, \sigma_{z}^{(j)}, j \in I$, and $c_{j}, c_{j}^{*}, j \in I, \mathfrak{A}(I)=\mathfrak{A} \cap \hat{\mathfrak{A}}(I)$ to be the subalgebra 
of $\mathfrak{U}$ generated by $\sigma_{x}^{(j)}, \sigma_{y}^{(j)}$, and $\sigma_{z}^{(j)}, j \in I$, and $\mathfrak{A}^{\mathrm{CAR}}(I)=\mathfrak{A}^{\mathrm{CAR}} \cap \hat{\mathfrak{U}}(I)$ to be the subalgebra of $\mathfrak{U}^{\text {CAR }}$ generated by $c_{j}$ and $c_{j}^{*}, j \in I$. We define $\hat{\mathfrak{A}}_{0}$ and $\mathfrak{U}_{0}^{\mathrm{CAR}}$ to be the union of $\hat{\mathfrak{U}}(I)$ and $\mathfrak{U}^{\mathrm{CAR}}(I)$ for all finite intervals $I$ of $\mathbf{Z}$, respectively. They are subalgebras of $\hat{\mathfrak{U}}$ and $\mathfrak{U}^{\mathrm{CAR}}$, and

$$
\begin{aligned}
\mathfrak{U}_{0}^{\mathrm{CAR}} & =\hat{\mathfrak{U}}_{0} \cap \mathfrak{U}^{\mathrm{CAR}}, \\
\mathfrak{U}_{0} & =\hat{\mathfrak{A}}_{0} \cap \mathfrak{U},
\end{aligned}
$$

where the latter is defined already in Sect. 2.

Let $\mathfrak{U}_{+}(I)$ denote $\mathfrak{U}_{+} \cap \mathfrak{U}(I)$, i.e. the set of $\Theta$-even elements of $\mathfrak{U}(I)$. It coincides with $\mathfrak{U}_{+}^{\mathrm{CAR}}(I)=\mathfrak{U}_{+} \cap \mathfrak{U}^{\mathrm{CAR}}(I)$ for any interval $I$ due to relations such as

$$
\begin{gathered}
\sigma_{\alpha}^{(j)} \sigma_{\beta}^{(j+n)}=d_{\alpha}^{(j)}\left(\prod_{k=j}^{j+n-1}\left(2 c_{k}^{*} c_{k}-1\right)\right) d_{\beta}^{(j+n)}, \\
d_{\alpha}^{(j)} d_{\beta}^{(j+n)}=\sigma_{\alpha}^{(j)}\left(\prod_{k=j}^{j+n-1} \sigma_{z}^{(k)}\right) \sigma_{\beta}^{(j+n)},
\end{gathered}
$$

where $(\alpha, \beta)=(x, x),(x, y),(y, x),(y, y)$ and

$$
d_{x}^{(l)}=c_{l}+c_{l}^{*}, \quad d_{y}^{(l)}=i\left(c_{l}-c_{l}^{*}\right) .
$$

Let $\mathscr{L}$ be the set of all derivations $\delta$ of $\hat{\mathfrak{A}}_{0}$ such that there exists an index set $\Xi=\{\alpha\}$, a finite interval $I_{\alpha}$ in $\mathbf{Z}$ for each $\alpha$ and $X_{\alpha}=X_{\alpha}^{*} \in \mathfrak{U}_{+}\left(I_{\alpha}\right)$ satisfying the following two conditions:

$$
\delta(A)=i \sum_{\alpha}\left[X_{\alpha}, A\right], \quad A \in \hat{\mathfrak{U}}_{0},
$$

(B) The set $\Xi_{j}$ of $\alpha \in \Xi$ such that $j \in I_{\alpha}$ is finite.

Proposition 5.1. The sum in (5.6) is a finite sum of non-zero elements in $\hat{\mathfrak{A}}_{0}$. The set $\mathscr{L}$ is a real Lie algebra of symmetric derivations on $\hat{\mathfrak{A}}_{0}$.

Proof. For $A \in \hat{\mathfrak{U}}(I)$, consider $I_{\alpha}$ such that $I \cap I_{\alpha}$ is empty. Then $\mathfrak{U}_{+}\left(I_{\alpha}\right)$ and $\mathfrak{U}(I)$ commute because $I \cap I_{\alpha}$ is empty and $\mathfrak{A}_{+}^{\mathrm{CAR}}\left(I_{\alpha}\right)$ and $\mathfrak{Y}^{\mathrm{CAR}}(I)$ commute for the same reason. Since $\mathfrak{U}_{+}\left(I_{\alpha}\right)=\mathfrak{U}_{+}^{\mathrm{CAR}}\left(I_{\alpha}\right)$ and $\hat{\mathfrak{U}}(I)$ is generated by $\mathfrak{U}(I)$ and $\mathfrak{U}^{\mathrm{CAR}}(I), A$ commutes with $X_{\alpha}$. Thus $\left[X_{\alpha}, A\right]=0$ unless $\alpha$ belongs to the finite set

$$
\left(\bigcup_{j \in I} \Xi_{j}\right)
$$

and hence the sum in (5.6) is finite and belongs to $\hat{\mathfrak{A}}_{0}$ for $A \in \hat{\mathfrak{U}}_{0}$.

Due to $X_{\alpha}^{*}=X_{\alpha}, \delta$ defined by (5.6) is a symmetric derivation. If $\delta_{1}$ is defined by (5.6) with $Y_{\beta}, \beta \in \Xi^{\prime}$, instead of $X_{\alpha}, \alpha \in \Xi$, then we have

$$
\left(\delta+\delta_{1}\right)(A)=i \sum_{v}\left[Z_{v}, A\right]
$$

where $v \in \Xi \cup \Xi^{\prime}$ and $Z_{\alpha}=X_{\alpha}$ for $\alpha \in \Xi, Z_{\beta}=Y_{\beta}$ for $\beta \in \Xi^{\prime}$,

$$
(c \delta)(A)=i \sum_{\alpha}\left[c X_{\alpha}, A\right]
$$

for any real $c$. Further,

$$
\left[\delta, \delta_{1}\right](A)=i \sum_{\mu}\left[W_{\mu}, A\right]
$$


where $\mu=(\alpha, \beta) \in \Xi \times \Xi^{\prime} \equiv \Xi^{\prime \prime}$ is restricted to the pair such that $I_{\alpha} \cap I_{\beta}$ is non-empty and

$$
W_{\mu}=i\left[X_{\alpha}, Y_{\beta}\right] \in \mathfrak{A}_{+}\left(I_{\alpha} \cup I_{\beta}\right),
$$

where $I_{\alpha} \cup I_{\beta}$ is again a finite interval and hence

$$
\begin{aligned}
\Xi_{(\alpha, \beta)}^{\prime \prime}= & \left\{(\alpha, \beta) ; j \in I_{\alpha}, k \in I_{\beta} \text { for some } k \in I_{\alpha}\right\} \\
& \cup\left\{(\alpha, \beta) ; j \in I_{\beta}, k \in I_{\alpha} \text { for some } k \in I_{\beta}\right\}
\end{aligned}
$$

is finite. Therefore $\mathscr{L}$ is a Lie algebra of symmetric derivations on $\hat{\mathfrak{A}}_{0}$. Q.E.D.

Proposition 5.2. A derivation $\delta \in \mathscr{L}$ is determined by its value on $B(h)$ for all $h \in \mathscr{H}$ with a finite number of non-zero components $h_{n}$.

Proof. First the finite linear span of $B(h)$ with such $h$ is the same as the finite linear span of $c_{j}$ and $c_{j}^{+}, j \in \mathbf{Z}$. By the linearity and the derivation property

$$
\delta\left(A_{1} \ldots A_{n}\right)=\sum_{j} A_{1} \ldots A_{j-1} \delta\left(A_{j}\right) A_{j+1} \ldots A_{n},
$$

the value of $\delta$ on $B(h)$ determines its value on $\mathfrak{A}_{0}^{\mathrm{CAR}}$.

Next we have

$$
\begin{gathered}
\delta(T)=i \sum_{\alpha}\left[X_{\alpha}, T\right]=b T, \\
b=i \sum_{\alpha}\left(X_{\alpha}-\Theta_{-}\left(X_{\alpha}\right)\right) \in \mathfrak{U}_{+}\left(I^{0}\right),
\end{gathered}
$$

where the sum can be restricted to $\alpha \in \Xi_{0}$, for example, and $I^{0}=\cup\left\{I_{\alpha} ; \alpha \in \Xi_{0}\right\}$. Note that, on $\mathfrak{U}\left(I_{\alpha}\right), \Theta_{-}=$id if $I_{\alpha}>0$ and $\Theta_{-}=\Theta$ if $I_{\alpha}<0$, so that $\Theta_{-}\left(X_{\alpha}\right)=X_{\alpha}$ in either case because $X_{\alpha} \in \mathfrak{A}_{+}\left(I_{\alpha}\right)$. For any $A \in \mathfrak{A}_{0}^{\text {CAR }}$, the relation $T \Theta_{-}(A) T=A$ implies

$$
\begin{aligned}
\delta(A)- & \Theta_{-} \delta\left(\Theta_{-}(A)\right)=\delta\left(T \Theta_{-}(A) T\right)-T \delta\left(\Theta_{-}(A)\right) T \\
& =\delta(T) \Theta_{-}(A) T+T \Theta_{-}(A) \delta(T)=b T \Theta_{-}(A) T+T \Theta_{-}(A) T T b T \\
& =b A+A \Theta_{-}(b) .
\end{aligned}
$$

On the other hand $\delta(1)=\delta\left(1^{2}\right)=1 \delta(1)+\delta(1) 1=2 \delta(1)$ implies $\delta(1)=0$ as is wellknown, and hence

$$
0=\delta(1)=\delta\left(T^{2}\right)=\delta(T) T+T \delta(T)=b T^{2}+T b T=b+\Theta_{-}(b) .
$$

Therefore (5.15) and (5.16) determine the commutator

$$
[b, A]=\delta(A)-\Theta_{-} \delta\left(\Theta_{-}(A)\right)
$$

for any $A \in \mathfrak{Q}_{0}^{\mathrm{CAR}}$ in terms of the value of $\delta$ on $\mathfrak{R}_{0}^{\mathrm{CAR}}$.

There exists a unique tracial state $\tau$ on $\mathfrak{A}^{\mathrm{CAR}}$, induced from the unique tracial state on full matrix algebras. Since

$$
\tau\left(U A U^{*}\right)=\tau\left(U^{*} U A\right)=\tau(A)
$$

for any $A \in \mathfrak{Q}^{\text {CAR }}$ and unitary $U$ due to the tracial property of $\tau$, we obtain from (3.2)

$$
\tau\left(\Theta_{-}(A)\right)=\tau(A)
$$


for any $A \in \mathfrak{U}^{\mathrm{CAR}}$. Then the formula (5.14b) implies

$$
\tau(b)=0 \text {. }
$$

The algebra $\operatorname{UPAR}^{\mathrm{CAR}}\left(I^{0}\right)$ is isomorphic to the full $2^{N} \times 2^{N}$ matrix algebra where $N$ is the cardinal number of the finite set $I^{0}$. Let $u_{i j}, i, j=1,2, \ldots, 2^{N}$ be its matrix unit. Any $A \in \mathfrak{U}^{\mathrm{CAR}}\left(I^{0}\right)$ can be written as

$$
b=2^{-N}\left(\sum_{k, j} u_{k j}\left[u_{j k}, b\right]+\tau(b) 1\right) .
$$

Therefore (5.17) and (5.20) uniquely determine $b \in \mathfrak{U}_{+}\left(I^{0}\right) \subset \mathfrak{U}_{0}^{\text {CAR }}$ in terms of the values of $\delta$ on $\mathfrak{U}_{0}^{\text {CAR }}$. Q.E.D.

Remark. In the above proof, we used an explicit form (5.14b) to show that $\tau(b)=0$. This can be avoided by using the following argument.

We consider the following linear functional on $\hat{\mathfrak{A}}$ :

$$
\hat{\tau}\left(A_{1}+A_{2} T\right)=\tau\left(A_{1}\right) \quad\left(A_{1}, A_{2} \in \mathfrak{A}^{\mathrm{CAR}}\right) .
$$

Then

$$
\hat{\tau}\left(\left(A_{1}+A_{2} T\right)^{*}\left(A_{1}+A_{2} T\right)\right)=\tau\left(A_{1}^{*} A_{1}+\Theta_{-}\left(A_{2}^{*}\right) \Theta_{-}\left(A_{2}\right)\right) \geqq 0
$$

and hence $\hat{\tau}$ is a state. Furthermore by (5.19) we obtain

$$
\begin{aligned}
& \hat{\tau}\left(\left(A_{1}+A_{2} T\right)\left(A_{1}+A_{2} T\right)^{*}\right)=\tau\left(A_{1} A_{1}^{*}+A_{2} A_{2}^{*}\right) \\
& \quad=\tau\left(A_{1}^{*} A_{1}+A_{2}^{*} A_{2}\right)=\tau\left(A_{1}^{*} A_{1}+\Theta_{-}\left(A_{2}^{*} A_{2}\right)\right) \\
& \quad=\hat{\tau}\left(\left(A_{1}+A_{2} T\right)^{*}\left(A_{1}+A_{2} T\right)\right) .
\end{aligned}
$$

This proves that $\hat{\tau}$ is tracial.

From the middle expression in (5.16), we obtain

$$
\tau(b)=\hat{\tau}(\delta(T) T)=\hat{\imath}(T \delta(T)+\delta(T) T) / 2=0 .
$$

This argument can be used whenever we know that $\delta(T)=b T$ with $b \in \mathfrak{A}^{\text {CAR }}$.

\section{Automorphism Group}

If all $X_{\alpha}$ in (5.3) are quadratic polynomials of $c_{j}$ and $c_{j}^{*}, j \in I_{\alpha}$, then $\delta(B(h))=B(\mathbf{X} h)$ for a skew symmetric linear operator $\mathbf{X}$ defined on $h \in \mathscr{H}$ with a finite number of nonzero components. Let $\mathscr{L}_{B}$ be the subset of $\mathscr{L}$ consisting of such derivations satisfying the following two conditions:

(a) $\mathbf{X}$ is bounded.

(b) $\left[\theta_{-}, \mathbf{X}\right]$ is in the trace class.

Here $\theta_{-}$is the operator defined by (3.22). have

Actually (b) is automatic and a stronger statement hold for $\delta \in \mathscr{L}$. Namely, we

$$
\begin{aligned}
& B\left(\left(\theta_{-} \mathbf{X} \theta_{-}-\mathbf{X}\right) h\right)=\Theta_{-} \delta\left(\Theta_{-}(B(h))\right)-\delta(B(h)) \\
& \quad=i \sum_{\alpha}\left(\Theta_{-}\left(\left[X_{\alpha}, \Theta_{-}(B(h))\right]\right)-\left[X_{\alpha}, B(h)\right]\right) \\
& \quad=i \sum_{\alpha}\left[\left(\Theta_{-}\left(X_{\alpha}\right)-X_{\alpha}\right), B(h)\right] .
\end{aligned}
$$


If 0 is not in the interval $I_{\alpha}$, then $\Theta_{-}\left(X_{\alpha}\right)=X_{\alpha}$ for $X_{\alpha} \in \mathfrak{U}_{+}\left(I_{\alpha}\right)$ and hence the sum is over $\alpha$ in a finite set $\Xi_{0}$. For each $\alpha \in \Xi_{0}, \Theta_{-}\left(X_{\alpha}\right)-X_{\alpha}$ is a quadratic expression of a finite number of $c_{j}$ and $c_{j}^{*}$ and hence

$$
i\left[\Theta_{-}\left(X_{\alpha}\right)-X_{\alpha}, B(h)\right]=B\left(\mathbf{x}_{\alpha} h\right),
$$

where $\mathbf{x}_{\alpha}$ is of a finite rank. Hence the following stronger property holds:

$$
\mathbf{Y} \equiv \theta_{-} \mathbf{X} \theta_{-}-\mathbf{X} \text { is of a finite rank . }
$$

This implies (b) due to $\left[\theta_{-}, \mathbf{X}\right]=\mathbf{Y} \theta_{-}$.

Proposition 6.1. The subset $\mathscr{L}_{B}$ is a Lie subalgebra of $\mathscr{L}$. For any $\delta \in \mathscr{L}_{B}$, there exists a one-parameter group $\exp \bar{\delta}$ t of automorphisms of $\hat{\mathfrak{A}}$ with the closure $\bar{\delta}$ of $\delta$ as its generator. It leaves the subalgebras $\mathfrak{A}^{\mathrm{CAR}}, \mathfrak{U}$ and $\mathfrak{U}_{+}$of $\mathfrak{\mathfrak { A }}$ invariant as sets.

Proof. If derivations $\delta_{1}$ and $\delta_{2}$ in $\mathscr{L}_{B}$ satisfy $\delta_{j}(B(h))=B\left(\mathbf{X}_{j} h\right)$, then $\delta=\left[\delta_{1}, \delta_{2}\right]$ satisfies $\delta(B(h))=B(\mathbf{X} h)$ with $\mathbf{X}=\left[\mathbf{X}_{1}, \mathbf{X}_{2}\right]$. The linear operator $\mathbf{X}$ satisfies (a) and (b), where we use the Jacobi identity for commutators as well as the ideal property of the trace class operators to obtain (b). Therefore $\mathscr{L}_{B}$ is a Lie subalgebra of $\mathscr{L}$.

Due to $X_{\alpha}^{*}=X_{\alpha}, \delta$ is a symmetric derivation and hence

$$
\begin{aligned}
B(\Gamma \mathbf{X} h) & =B(\mathbf{X} h)^{*}=\delta(B(h))^{*}=\delta\left(B(h)^{*}\right) \\
& =\delta(B(\Gamma h))=B(\mathbf{X} \Gamma h)
\end{aligned}
$$

from which we obtain

$$
[\mathbf{X}, \Gamma]=0 .
$$

Since $\delta(1)=0$, the CAR implies

$$
\begin{aligned}
0 & =\delta\left(\left[B\left(h_{1}\right)^{*}, B\left(h_{2}\right)\right]_{+}\right)=\left[B\left(\mathbf{X} h_{1}\right)^{*}, B\left(h_{2}\right)\right]_{+}+\left[B\left(h_{1}\right)^{*}, B\left(\mathbf{X} h_{2}\right)\right]_{+} \\
& =\left(\mathbf{X} h_{1}, h_{2}\right)+\left(h_{1}, \mathbf{X} h_{2}\right)
\end{aligned}
$$

for all $h_{1}, h_{2}$ with a finite number of components. Therefore $X$ is skew symmetric:

$$
\mathbf{X}^{*}=-\mathbf{X} \text {. }
$$

By (6.2) and (6.4),

$$
U_{t}=e^{\mathbf{X} t}
$$

is a unitary operator commuting with $\Gamma$ and hence defines a Bogoliubov automorphism $\alpha_{U_{t}}$ of $\mathfrak{Q}^{\mathrm{CAR}}$ satisfying

$$
\alpha_{U_{t}}(B(h))=B\left(U_{t} h\right) .
$$

The generator of $\alpha_{U_{t}}$ then coincides with $\delta$ on $B(h)$ and hence on $\mathfrak{I}_{0}^{\mathrm{CAR}}$.

By the condition (b),

$$
\mathbf{Y}=\theta_{-} \mathbf{X} \theta_{-}-\mathbf{X}=\left[\theta_{-}, \mathbf{X}\right] \theta_{-}
$$

is in the trace class and satisfies

$$
\Gamma \mathbf{X}^{*} \Gamma=-\mathbf{Y}
$$


due to $\left[\Gamma, \theta_{-}\right]=0,(6.4)$ and (6.6). There exists an element $Y \in \mathfrak{O}_{+}^{\text {CAR }}$ called the bilinear Hamiltonian $\left(Y=(1 / 2)(B, \mathbf{Y} B)\right.$ in the notation of [1]) satisfying $Y^{*}=-Y$ (due to $\mathbf{Y}^{*}=-\mathbf{Y}$ ) and

$$
[Y, B(h)]=B(\mathbf{Y} h) \text {. }
$$

By (7.12) of $[1]$ and $\theta_{-} \mathbf{Y} \theta_{-}=-\mathbf{Y}$, it also satisfies

$$
\Theta_{-}(Y)=-Y \text {. }
$$

Using the expansional formalism in [2], we define

$$
W_{t}=1+\sum_{n=1}^{\infty} \int_{0}^{t} \ldots \int_{0}^{t_{n-1}} Y_{t_{n}} \ldots Y_{t_{1}} d t_{n} \ldots d t_{1}
$$

where $Y_{t} \equiv \alpha_{U_{t}}(Y)$. By using Eqs. (2.17), (2.18), Propositions 3 and 4 of [2] as well as the property $Y^{*}=-Y$, we see that $W_{t}$ is unitary. By Theorem 2 of [2], $W_{t}$ satisfies the cocycle equation:

$$
W_{s} \alpha_{U_{s}}\left(W_{t}\right)=W_{s+t} .
$$

Therefore

$$
\beta_{t}(A)=W_{t} \alpha_{U_{t}}(A) W_{t}^{*}, \quad t \in \mathfrak{A}^{\mathrm{CAR}}
$$

defines a continuous one-parameter group of automorphisms of $\mathfrak{A}^{\mathrm{CAR}}$.

Using (4.11) of [2], we obtain for $V_{t}=e^{\theta_{-} X \theta_{-} t}=\theta_{-} U_{t} \theta_{-}$

$$
\begin{aligned}
& (d / d t) \beta_{-t}\left(B\left(V_{t} h\right)\right)=\beta_{-t}(d / d s) \beta_{-s}\left(B\left(V_{t+s} h\right)\right)_{s=0} \\
& \quad=\beta_{-t}\left\{-\left.(d / d s) \alpha_{U_{s}}\left(B\left(V_{t} h\right)\right)\right|_{s=0}-\left[Y, B\left(V_{t} h\right)\right]+B\left(d V_{t} h / d t\right)\right\} \\
& \quad=\beta_{-t}\left\{-B\left(\mathbf{X} V_{t} h\right)-B\left(\mathbf{Y} V_{t} h\right)+B\left(\theta_{-} \mathbf{X} \theta_{-} V_{t} h\right)\right\}=0 .
\end{aligned}
$$

Therefore $\beta_{-t}\left(B\left(V_{t} h\right)\right)$ is constant in $t$. Since its value at $t=0$ is $B(h)$, we have

$$
\beta_{t}(B(h))=B\left(V_{t} h\right)=\alpha_{V_{t}}(h)=\Theta_{-} \alpha_{U_{t}} \Theta_{-}(B(h)) .
$$

Thus we have obtained the following formula:

$$
W_{t} \alpha_{U_{t}}(A) W_{t}^{*}=\alpha_{V_{t}}(A)=\Theta_{-} \alpha_{U_{t}} \Theta_{-}(A)
$$

for $A=B(h)$ and hence for all $A \in \mathfrak{Q}^{\mathrm{CAR}}$.

By (6.12) and (6.13), we obtain

$$
\Theta_{-}\left(W_{t}\right)=1+\sum_{n=1}^{\infty} \int_{0}^{t} \ldots \int_{0}^{t_{n-1}}\left(-Y_{t_{n}}^{\prime}\right) \ldots\left(-Y_{t_{1}}^{\prime}\right) d t_{n} \ldots d t_{1}
$$

where we have used the relation

$$
\Theta_{-}\left(Y_{t}\right)=\Theta_{-} \alpha_{U_{t}} \Theta_{-}\left(\Theta_{-}(Y)\right)=\alpha_{V_{t}}(-Y)
$$

and notation

$$
Y_{t}^{\prime}=\alpha_{V_{t}}(Y)=W_{t} \alpha_{U_{t}}(Y) W_{t}^{*} .
$$

The quantity $-Y_{t}^{\prime}$ corresponds to $(Y *(-Y))(t)$ of (3.7) in [2] with $B=Y$ and $A=-Y$ and hence

$$
\Theta_{-}\left(W_{t}\right) W_{t}=1
$$

due to $A+B=0$ in (3.10) of [2].

We now define the extension $\alpha_{t}$ of $\alpha_{U_{t}}$ from $\mathfrak{A}^{\mathrm{CAR}}$ to $\hat{\mathfrak{A}}$ by

$$
\alpha_{t}\left(A_{1}+A_{2} T\right)=\alpha_{U_{t}}\left(A_{1}\right)+\alpha_{U_{t}}\left(A_{2}\right) T W_{t} .
$$


To show that $\alpha_{t}$ is indeed a $*$ automorphism, we have to see that relations $T^{2}=1$, $T^{*}=T$ and $T A T=\Theta_{-}(A)$ for $A \in \mathfrak{U}^{\mathrm{CAR}}$ are preserved, namely it is enough to check the following relations:

$$
\begin{gathered}
T W_{t} T W_{t}=1, \\
W_{t}^{*} T=T W_{t}, \\
T W_{t} \alpha_{U_{t}}(A) T W_{t}=\alpha_{U_{t}}\left(\Theta_{-}(A)\right) .
\end{gathered}
$$

Equation (6.24) holds by (6.22) and $\Theta_{-}\left(W_{t}\right)=T W_{t} T$. Because $W_{t}$ is unitary, (6.24) implies $W_{t}^{*}=T W_{t} T$ and this implies (6.25). By (6.25) and (6.18), we obtain

$$
\begin{aligned}
T W_{t} \alpha_{U_{t}}(A) T W_{t} & =T W_{t} \alpha_{U_{t}}(A) W_{t}^{*} T \\
& =T\left\{\Theta_{-} \alpha_{U_{t}} \Theta_{-}(A)\right\} T=\alpha_{U_{t}} \Theta_{-}(A)
\end{aligned}
$$

which shows (6.26).

In the notation of [1], we have

$$
\alpha_{U_{t}}(B, \mathbf{Y} B)=\left(B, U_{t} \mathbf{Y} U_{-t} B\right)
$$

by (7.12) of [1]. It is an entire analytic function of a complex number $t$, because $U_{z}=e^{z \mathbf{X}}$ is an entire analytic function of $z$ (values are bounded operators), $\mathbf{Y}$ is in the trace class and $(B, L B)$ is linear and continuous in $L$ with the bound

$$
\left\|Y_{z}\right\| \leqq(1 / 2)\|\mathbf{Y}\|_{\text {tr }} e^{2\||\mathbf{X} \|| \operatorname{Im} z \mid} .
$$

Therefore $W_{t}$ of (6.13) has an entire analytic extension:

$$
W_{z}=1+\sum_{n=1}^{\infty} z^{n} \int_{0}^{1} \int_{0}^{t_{1}} \ldots \int_{0}^{t_{n-1}} Y_{z t_{n}} \ldots Y_{z t_{1}} d t_{n} \ldots d t_{1} .
$$

In particular, $T$ is in the domain of the generator of $\alpha_{t}$. Since the generator of $\alpha_{t}$ is a symmetric derivation coinciding with $\delta$ on $\mathfrak{Q}^{\mathrm{CAR}}$, it must be an extension of $\delta$ by the uniqueness result given in Proposition 5.2 (see Remark after Proof of Proposition 5.2).

Since $\alpha_{z}(B(h))=B\left(e^{z \mathbf{X}} h\right)$ and $\alpha_{z}(T)=T W_{z}$ are entire analytic, all elements of $\hat{\mathfrak{A}}_{0}$ are entire analytic elements of $\delta$. Thus the condition (B2) of Theorem 3.2.50 of [8] is satisfied. Since the generator of $\alpha_{t}$ satisfies the condition (C1) there, it is satisfied by $\delta$ which is a restriction of the generator to $\hat{\mathfrak{A}}_{0}$. The condition (A1) is also satisfied with $D(\delta)=\hat{\mathfrak{A}}_{0}$. Therefore the closure of $\delta$ is exactly the generator of $\alpha_{t}$ by Theorem 3.2.50 of [8].

Since $\alpha_{t}$ maps $B(h)$ to $B\left(U_{t} h\right)$ and $T$ to $T W_{t}$ with $W_{t} \in \mathfrak{A}_{+}$, the subalgebras $\mathfrak{A}^{\mathrm{CAR}}$, $\mathfrak{U}$ and $\mathfrak{U}_{+}$are invariant under $\alpha_{t}$ as sets. Q.E.D.

Remark to Theorem 6.1. Except for the first statement (that $\mathscr{L}_{B}$ is a Lie algebra), Theorem 6.1 holds for $\delta$ defined on $\hat{\mathfrak{A}}_{0}$ such that $i X$ is analytic on the set of all $h \in \mathscr{H}$ with a finite number of non-zero components. The proof is the same because $U_{t}=e^{\widehat{\mathbf{X}} t}$ will have the same property as $U_{t}$ of (6.7), $\delta$ is analytic on $B(h)$ in $\mathfrak{A}_{0}$ (i.e. when $h$ has a finite number of non-zero components),

$$
G(t) \equiv \sup _{-t \leqq s \leqq t}\left\|e^{i \widehat{\mathbf{x}} t} E\right\|
$$


is finite for small $t \geqq 0$ and for the (finite dimensional) projection $E$ on a finite interval of $\mathbf{Z}$ (projection considered in the Hilbert space $\mathscr{H}=l_{2}(\mathbf{Z}) \oplus l_{2}(\mathbf{Z})$ ) supporting the operator $\mathbf{Y}$, and the estimate (6.29) holds with $G(|\operatorname{Im} z|)^{2}$ replacing $e^{2|| \mathbf{X}|||\operatorname{Im} z|}$.

Proposition 6.2. The set of automorphisms

$$
\left.G_{0} \equiv\left\{\exp \bar{\delta} ; \delta \in i \mathfrak{g}_{0}\right\}\right|_{\mathscr{W} \mathrm{CAR}}
$$

(restricted to $\mathfrak{A}^{\mathrm{CAR}}$ ) is maximal abelian in the sense that any automorphism of $\mathfrak{P}^{\mathrm{CAR}}$ commuting with all elements of $G_{0}$ is in the closure of $G_{0}$ (relative to the pointwise convergence).

Proof. Let $E$ be the spectral projection of $\mathbf{K}$ for its positive spectrum, namely

$$
(E h)(\theta)=E(\theta) h, E(\theta)=(2 k(\theta))^{-1}(K(\theta)+k(\theta)) .
$$

Due to $\Gamma K \Gamma=-K$, we have

$$
\Gamma E \Gamma=1-E .
$$

Thus $E$ is a basis projection in the terminology of [1] and [6].

Let $M$ be the abelian von Neumann algebra of all bounded multiplication operators in $\theta$-representation on the space $E \mathscr{H}$ (rather than $\mathscr{H}$ ). It is then maximal abelian, as $E(\theta)$ is one-dimensional for each $\theta$.

For the Proof of Proposition 6.2, we need the following Lemma describing $\mathfrak{g}_{0}$ exactly.

Lemma 6.3. The derivations $\delta$ in $i \mathrm{~g}_{0}$ are exactly those of the following form:

$$
\begin{gathered}
\delta(B(h))=B(\hat{\delta} h),(\hat{\delta} h)(\theta)=\hat{\delta}(\theta) h(\theta), \\
\hat{\delta}(\theta)=i \delta_{1}(\theta) 1+i \delta_{2}(\theta) K(\theta),
\end{gathered}
$$

where $\delta_{1}$ and $\delta_{2}$ are any Laurent polynomial of $e^{i \theta}$ such that $\delta_{1}$ is a real odd function of $\theta$ and $\delta_{2}$ is a real even function of $\theta$ :

$$
\begin{gathered}
\overline{\delta_{1}(\theta)}=\delta_{1}(\theta), \quad \overline{\delta_{2}(\theta)}=\delta_{2}(\theta), \\
\delta_{1}(-\theta)=-\delta_{1}(\theta), \quad \delta_{2}(-\theta)=\delta_{2}(\theta) .
\end{gathered}
$$

Proof of Lemma. By the beginning part of the proof of Proposition 6.1, $\Gamma \hat{\delta} \Gamma=\hat{\delta}$ and $\hat{\delta}^{*}=-\hat{\delta}$. If $\hat{\delta}$ is of the form given by (6.34) and (6.35), then $\hat{\delta}^{*}=-\hat{\delta}$ implies (6.36) and (3.32) for $\Gamma$ implies (6.37).

Since

$$
k D k=k^{2} D+(1 / 2)\left(\partial k^{2} / \partial \theta\right),
$$

the formula for $H_{j}$ and $S_{j}$ shows that $\hat{\delta}$ s for $H_{j}$ and $S_{j}$ are in fact of the form given by (6.34) and (6.35) with $\delta_{1}$ and $\delta_{2}$ Laurent polynomials of $e^{i \theta}$. Since $k^{2}$ is at most of second degree and $D$ does not change degrees, we can find the exact degrees as follows: 
For $\gamma \neq \pm 1$

$\begin{array}{ccc} & \text { degree } & \text { coefficient } \\ h_{2 n}(\theta) & 2 n & 2^{2 n+1} J\left(\gamma^{2}-1\right)^{n}(2 n) ! \\ h_{2 n+1}(\theta) & 2 n+2 & -i 2^{2 n+2} J\left(\gamma^{2}-1\right)^{n+1}(2 n+1) ! \\ s_{2 n}(\theta) & 2 n+1 & i 2^{2 n+1}\left(\gamma^{2}-1\right)^{n}(2 n) ! \\ s_{2 n+1}(\theta) & 2 n+1 & -2^{2 n+2}\left(\gamma^{2}-1\right)^{n}(2 n+1) !\end{array}$

For $\gamma^{2}=1$ and $\lambda \neq 0$,

$$
\begin{array}{ccc}
h_{2 n}(\theta) & n & 2^{4 n+1} J \lambda^{n} n !^{2} \\
h_{2 n+1}(\theta) & n+1 & -i 2^{4 n+4} J \lambda^{n+1} n !(n+1) ! \\
s_{2 n}(\theta) & n+1 & i 2^{4 n+1} \lambda^{n} n !(n+1) ! \\
s_{2 n+1}(\theta) & n+1 & 2^{4 n+2} \lambda^{n}(n+1) !^{2}
\end{array}
$$

The case $(\lambda, \gamma)=(0, \pm 1)$ has been excluded from the beginning (see Sect. 2).

In addition, $h_{2 n}(\theta)$ and $s_{2 n+1}(\theta)$ are even functions of $\theta$ while $h_{2 n+1}(\theta)$ and $s_{2 n}(\theta)$ are odd functions of $\theta$. Therefore, by taking the real linear combination, we obtain any real odd polynomial of $e^{i \theta}$ for $\delta_{1}$ and any real even polynomial of $e^{i \theta}$ for $\delta_{2}$ (even-odd as a function of $\theta$ ). Note that a real even function which is of the highest degree $k$ as a Lausent polynomial of $e^{i \theta}$ is unique up to the addition of lower degree polynomials and a constant coefficient (for example $\cos k \theta$ ) and a real odd function which is of the highest degree $k$ is also unique in the same sense (for example $\sin k \theta$ ). This proves Lemma 6.3.

We remark that if $\gamma^{2}=1, \lambda \neq 0$, then the $S$ 's become redundant.

We now resume the proof of Proposition 6.2. By Stone-Weierstrass theorem, any periodic continuous function of $\theta$ can be approximated by Laurent polynomials of $e^{i \theta}$. The closure of $G_{0}$ in Aut $\mathfrak{H}^{\mathrm{CAR}}$ contains all Bogoliubov automorphisms $\alpha_{U}$ with $U=e^{\mathbf{X}}$ and

$$
(\mathbf{X} h)(\theta)=\hat{X}(\theta) h(\theta), \quad \hat{X}(\theta)=i X_{1}(\theta) 1+i X_{2}(\theta) K(\theta),
$$

where $X_{1}$ is any odd real periodic continuous function of $\theta$ and $X_{2}$ is any even real periodic continuous function. Since $\|B(h)\|=\|h\|$, by using approximation in strong operator topology on $\mathscr{H}$, closure of $G_{0}$ in Aut $\mathfrak{H}^{\mathrm{CAR}}$ contains $e^{\mathbf{X}}$ with any even real periodic $L^{\infty}$-function $X_{1}$ and any even real periodic measurable function $X_{2}$ such that $X_{2}(\theta) k(\theta)$ is essentially bounded. Then $(U k)(\theta)=\hat{U}(\theta) h(\theta)$ with UE exhausting unitary elements of $M$. Now the maximal abelian property of $G_{0}$ follows from the maximal abelian property of $M$ on $E \mathscr{H}$ by Theorem 2 of Kishimoto in [9].

Proposition 6.4. The subgroup

$$
\hat{G}_{0}=\left\{\exp \bar{\delta} ; \delta \in i \mathfrak{g}_{0}\right\}
$$

of Aut $\hat{\mathcal{A}}$ is abelian.

Proof. By Proposition 6.2, we already know that the action of $\hat{G}_{0}$ on $\mathfrak{H}_{0}$ is abelian. Therefore this Proposition follows from the following uniqueness result about 
extension of automorphisms because $\alpha_{1} \alpha_{2}$ and $\alpha_{2} \alpha_{1}$ always coincide as automorphisms of $\hat{\mathfrak{A}}^{\mathrm{CAR}}$ for two automorphisms in $\hat{G}_{0}$ and hence they must coincide also on $\hat{\mathfrak{A}}$ as $\hat{G}_{0}$ is connected.

Lemma 6.5. Let $\left\{\alpha_{t}\right\},\left\{\beta_{t}\right\}, 0 \leqq t \leqq 1$, be two continuous families of automorphisms of $\hat{\mathfrak{A}}$ such that they leave $\mathfrak{Q}^{\mathrm{CAR}}$ invariant as a set and

$$
\alpha_{t}(T) T \in \mathfrak{A}^{\mathrm{CAR}}, \quad \beta_{t}(T) T \in \mathfrak{A}^{\mathrm{CAR}} .
$$

If $\alpha_{t}$ and $\beta_{t}$ coincide on $\mathfrak{2}^{\mathrm{CAR}}$ and if $\alpha_{0}=\beta_{0}$, then $\alpha_{t}=\beta_{t}$ for all $t$.

Proof. It is enough to show

$$
\alpha_{t}(T)=\beta_{t}(T)
$$

For any $A \in \mathfrak{A}^{\mathrm{CAR}}$,

$$
\begin{aligned}
& \alpha_{t}(T) A \alpha_{t}(T)=\alpha_{t}\left(T \alpha_{-t}(A) T\right)=\alpha_{t} \Theta_{-} \alpha_{-t}(A), \\
& \beta_{t}(T) A \beta_{t}(T)=\beta_{t}\left(T \beta_{-t}(A) T\right)=\beta_{t} \Theta_{-} \beta_{-t}(A) .
\end{aligned}
$$

Since $\alpha_{t}=\beta_{t}$ on $\mathfrak{A}^{\mathrm{CAR}}$, we obtain

$$
\beta_{t}(T) \alpha_{t}(T) A \alpha_{t}(T) \beta_{t}(T)=\beta_{t} \Theta_{t} \beta_{-t} \alpha_{t} \Theta_{-} \alpha_{-t}(A)=A .
$$

Since

$$
\begin{aligned}
& \alpha_{t}(T)^{2}=\alpha_{t}\left(T^{2}\right)=\alpha_{t}(1)=1, \\
& \beta_{t}(T)^{2}=\beta_{t}\left(T^{2}\right)=\beta_{t}(1)=1,
\end{aligned}
$$

(6.44) implies

$$
\left[\beta_{t}(T) \alpha_{t}(T), A\right]=0
$$

By (6.41)

$$
\beta_{t}(T) \alpha_{t}(T)=\left(\beta_{t}(T) T\right)\left(T \alpha_{t}(T)\right)=\left(\beta_{t}(T) T\right)\left(\alpha_{t}(T) T\right)^{*} \in \mathfrak{A}^{\mathrm{CAR}} .
$$

Therefore $\beta_{t}(T) \alpha_{t}(T)$ is a scalar operator $\lambda 1$. By multiplying $\alpha_{t}(T)$ and using $(6.45 \mathrm{a})$, we have

$$
\beta_{t}(T)=\lambda \alpha_{t}(T)
$$

for some complex number $\lambda$. By (6.45a) and (6.45b), $\lambda^{2}=1$ and hence

$$
\beta_{t}(T)= \pm \alpha_{t}(T) \text {. }
$$

By continuity, the sign is common for all $t$ and, since it is +1 for $t=0$ by assumption, we obtain (6.42) for all $t$. Q.E.D.

\section{Conclusion}

Proofs of Theorems in Sect. 2 are essentially given in the preceding sections. We summarize it below.

The commutativity of all $H_{k, j}$ and $S_{k, j}$ as derivations on $\mathfrak{A}^{\mathrm{CAR}}$ are immediate from concrete expressions given to them in Propositions 4.1, 4.2, 4.4, and 4.5. Since 
these derivations are of the class $\mathscr{L}$ treated in Proposition 5.2, the uniqueness of extension from $\mathfrak{A}^{\mathrm{CAR}}$ to $\hat{\mathfrak{A}}$ shown in Proposition 5.2 proves the commutativity of these derivations on $\mathfrak{A}$ and hence on $\mathfrak{A}$. This proves Theorems 1 and 2.

Since these derivations are of the form given in Lemma 6.3 (as follows from explicit forms given by Propositions 4.4 and 4.5), $H_{k, j}$ and $S_{k, j}$ are contained in $g_{0}$ by Lemma 6.3. This proves Theorem 3.

The second half of Theorem 4 is Proposition 6.4. The first half of Theorem 4 will follow from Remark to Proposition 6.1 if we prove that all $h \in \mathscr{H}$ with a finite number of non-zero components are analytic vectors of any $X \in \mathfrak{g}_{1}$.

For such an $h$,

$$
h(\theta)=\sum_{|j| \leqq m} h_{j} e^{i j \theta},
$$

where each $h_{j}$ is a constant of $\theta$. Let $\alpha=\max _{j}\left\|h_{j}\right\|_{\mathscr{H}}$. Any $X \in \mathfrak{g}_{1}$ is of the form

$$
X=l_{1}(\theta) D+l_{2}(\theta), \quad l_{p}(\theta)=\sum_{|j| \leqq k} l_{p j} e^{i j \theta},
$$

where $p=1,2$, each $l_{p j}$ is a $2 \times 2$ matrix, constant of $\theta$, and $D=d / d \theta$. Let $\beta=\max _{p, j}\left\|l_{p j}\right\|$. Then, $X^{n} h$ is a sum of $(4 k+2)^{n}(2 m+1)$ terms $((4 h+2)$ is the number of terms in $X$ and $(2 m+1)$ is the number of terms in $h)$ of the form

$$
\left\{e^{i j_{n} \theta}(D) \ldots e^{i j_{2} \theta}(D) e^{i j_{1} \theta}(D) e^{i j \theta}\right\} L_{n} \ldots L_{1} h_{j},
$$

where $|j| \leqq m,\left|j_{l}\right| \leqq k$ for all $l,(D)$ is either $D$ or 1 , and each $L_{l}$ is $l_{1 j_{l}}$ or $l_{2 j_{l}}$. The expression in the parenthesis of (7.3) has its absolute value bounded by

$$
|j|\left|j+j_{1}\right|\left|j+j_{1}+j_{2}\right| \ldots\left|j+j_{1}+\ldots+j_{n-1}\right| \leqq \prod_{l=0}^{n-1}(m+l k) .
$$

We also have

$$
\left\|L_{n} \ldots L_{1} h_{j}\right\| \leqq \beta^{n} \alpha
$$

These estimates imply

$$
\left\|X^{n} h\right\| \leqq(4 k+2)^{n} \beta^{n}(2 m+1) \alpha \prod_{l=0}^{n-1}(m+l k) .
$$

Hence

$$
\sum z^{n}\left\|X^{n} h\right\| / n !
$$

is convergent if

$$
|z|(4 k+2) \beta k<1 .
$$

Therefore, any $h$ is an analytic vector of $X$.

Proposition 6.2 leads to an obvious conjecture that $G_{0}$ might be maximal abelian on $\mathfrak{A}$. 


\section{References}

1. Araki, H.: On quasifree states of CAR and Bogoliubov automorphism. Publ. RIMS Kyoto Univ. 6, 385-442 (1970)

2. Araki, H.: Expansional in Banach algebras. Annal. Sci. École Normale Supérieure, Ser. 4, 6, 67-84 (1973)

3. Araki, H.: On the XY-model on two-sided infinite chain. Publ. RIMS Kyoto Univ. 20, 277-296 (1984)

4. Araki, H.: Ground states of the XY-model. Commun. Math. Phys. 101, 213-245 (1985)

5. Araki, H., Matsui, T.: Analyticity of ground states of the XY-model. Lett. Math. Phys. 11, 87-94 (1986)

6. Araki, H.: Bogoliubov automorphisms and Fock representation of canonical anticommutation relations. Contemp. Math. 62, 23-141 (1987)

7. Barouch, E., Fuchssteiner, B.: Master symmetries and similarity equations. Stud. Appl. Math. 73, 221-237 (1985)

8. Bratteli, O., Robinson, D.W.: Operator algebras and quantum statistical mechanics. Berlin, Heidelberg, New York: Springer 1979

9. Kishimoto, A.: On invariant states and the commutant of a group of quasifree automorphisms of the CAR algebra. Rep. Math. Phys. 15, 21-26 (1979)

Communicated by A. Jaffe

Received April 3, 1990 